\title{
Tracking the clonal origin of lethal prostate cancer
}

\author{
Michael C. Haffner, ${ }^{1}$ Timothy Mosbruger, ${ }^{1}$ David M. Esopi, ${ }^{1}$ Helen Fedor, ${ }^{2}$ Christopher M. Heaphy, ${ }^{2}$ \\ David A. Walker, ${ }^{1}$ Nkosi Adejola, ${ }^{1}$ Meltem Gürel, ${ }^{1}$ Jessica Hicks, ${ }^{2}$ Alan K. Meeker, ${ }^{1,2,3}$ \\ Marc K. Halushka, ${ }^{2}$ Jonathan W. Simons, ${ }^{4}$ William B. Isaacs, ${ }^{1,2,3}$ Angelo M. De Marzo, 1,2,3 \\ William G. Nelson, ${ }^{1,2,3}$ and Srinivasan Yegnasubramanian ${ }^{1}$
}

${ }^{1}$ Sidney Kimmel Comprehensive Cancer Center, ${ }^{2}$ Department of Pathology, and ${ }^{3}$ Brady Urological Institute, Johns Hopkins School of Medicine, Baltimore, Maryland, USA. ${ }^{4}$ Prostate Cancer Foundation, Santa Monica, California, USA.

\begin{abstract}
Recent controversies surrounding prostate cancer overtreatment emphasize the critical need to delineate the molecular features associated with progression to lethal metastatic disease. Here, we have used whole-genome sequencing and molecular pathological analyses to characterize the lethal cell clone in a patient who died of prostate cancer. We tracked the evolution of the lethal cell clone from the primary cancer to metastases through samples collected during disease progression and at the time of death. Surprisingly, these analyses revealed that the lethal clone arose from a small, relatively low-grade cancer focus in the primary tumor, and not from the bulk, higher-grade primary cancer or from a lymph node metastasis resected at prostatectomy. Despite being limited to one case, these findings highlight the potential importance of developing and implementing molecular prognostic and predictive markers, such as alterations of tumor suppressor proteins PTEN or p53, to augment current pathological evaluation and delineate clonal heterogeneity. Furthermore, this case illustrates the potential need in precision medicine to longitudinally sample metastatic lesions to capture the evolving constellation of alterations during progression. Similar comprehensive studies of additional prostate cancer cases are warranted to understand the extent to which these issues may challenge prostate cancer clinical management.
\end{abstract}

\section{Introduction}

Prostate cancer is the most frequently diagnosed malignancy and second leading cause of cancer-specific deaths in men in the United States (1). Clinically, prostate cancer is highly heterogeneous; manifestations vary from indolent localized tumors to widespread metastases. Given recent controversies surrounding overtreatment of prostate cancer, there is a critical need to understand the features of the primary tumor that are associated with progression to lethal disease $(2,3)$.

Primary prostate cancers often harbor multiple morphologically and clonally distinct tumor foci (4-6). Despite the multifocal and multiclonal heterogeneity of primary prostate tumors, most distant metastases from different anatomic sites in the same patient share the majority of genetic alterations, which suggests a monoclonal origin of lethal metastatic cells $(7,8)$. Therefore, identifying the characteristics of the primary cancer lesion that ultimately can give rise to the lethal metastatic cell clone is of great interest. Studying the full spectrum of prostate cancer presentation and progression would require longitudinal, integrated analysis of the primary cancer and matched metachronous metastases sampled during disease progression and at death (9). Perhaps due to the protracted natural history of prostate cancer, such a study has not been conducted thus far.

Here we present the case of a man with lethal metastatic prostate cancer for whom, through longitudinal sampling and comprehensive genomic and pathological analysis, we identified the constellation of genomic alterations that characterized the lethal

Conflict of interest: Angelo M. De Marzo was employed at Predictive Biosciences Inc. during a portion of these studies. No funding or other support was provided by Predictive Biosciences Inc. for any of the work in this manuscript.

Citation for this article: J Clin Invest. 2013;123(11):4918-4922. doi:10.1172/JCI70354. metastatic cell clone and traced its origin back to a specific lesion in the primary cancer.

\section{Results and Discussion}

The subject was diagnosed with prostate adenocarcinoma at age 47 years. His entire primary tumor and a single involved lymph node metastasis was initially removed by radical prostatectomy, but an elevated PSA level 5 years after surgery suggested systemic disease and prompted therapy with an investigational prostate cancer vaccine (GVAX; ref. 10), androgen ablation, systemic chemotherapy, and localized radiation (Supplemental Information and Supplemental Figure 1; supplemental material available online with this article; doi:10.1172/JCI70354DS1). Despite these interventions, 17 years after initial presentation, the patient succumbed to overwhelming castrate-resistant prostate cancer at 64 years of age. After death, tissues from 7 metastatic sites were procured by rapid autopsy. To define the genetic features of the cell clone that gave rise to the lethal tumor burden, we performed whole-genome sequencing on 3 anatomically distinct autopsy metastases - M5 (liver), M38 (perigastric lymph node), and M40 (lung) - and germline DNA, with average sequencing coverage exceeding 50× (Supplemental Table 1 and ref. 11). We identified 85 coding mutations and 226 structural rearrangements (19 [8.4\%] interchromosomal and 207 [91.6\%] intrachromosomal) that were common to all 3 metastatic sites (Figure 1 and Supplemental Tables 2 and 3). None of the metastases harbored rearrangements involving TMPRSS2, ERG, or other ETS transcription factors. All 3 metastases also shared widespread copy number alterations (Supplemental Figure 2 and Supplemental Table 4). A >60-fold amplification of the $A R$ locus was present in all distant hormonerefractory metastases (Supplemental Figure 3 and Supplemental Table 4). Although there was some genetic heterogeneity among 


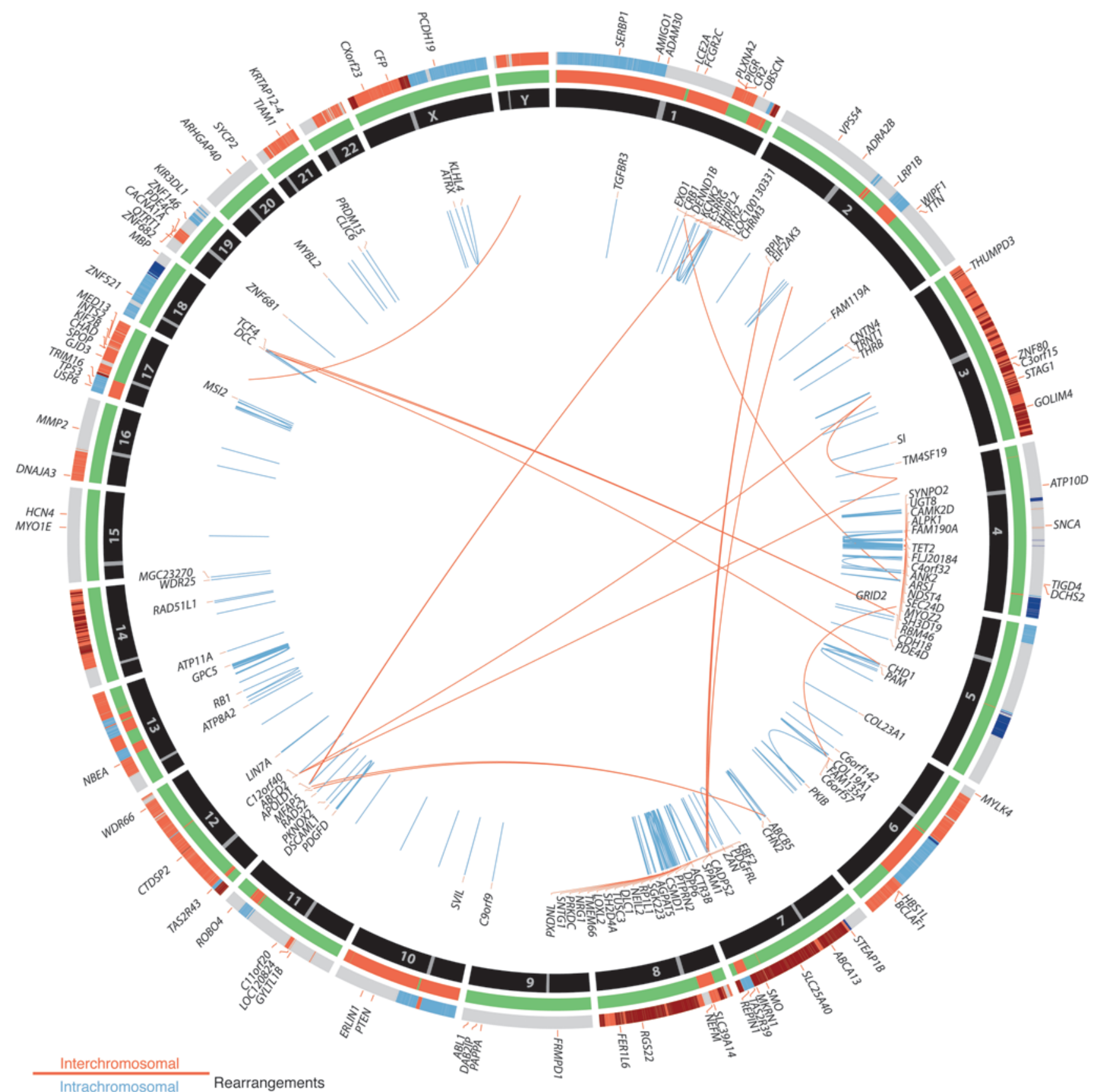

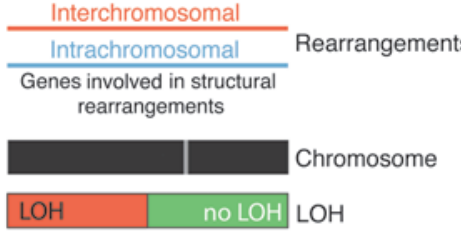

\begin{tabular}{l|l|l|l|l|}
\hline$>4$ & 2 & 0.5 & Copy number
\end{tabular} Genes containing coding SNVs

\section{Figure 1}

Common genomic consensus alterations found in 3 distant metastases (M5, M38, and M40) by whole-genome sequencing are plotted. Genes with nonsynonymous single nucleotide variants (SNVs) and indels in coding sequences are indicated in the outermost circle. Copy number alterations are color-coded and shown in the adjacent circle. Regions of high copy number gain $(>4$; dark red), and significant copy number loss ( $<0.5$; blue) are indicated. LOH of individual regions (orange) is indicated in the middle circle. Arcs in the interior connect regions of structural rearrangements (blue, intrachromosomal; orange, interchromosomal), and associated genes are shown in the inner circle. metastases (Supplemental Figure 2), the high number of shared alterations indicated a monoclonal origin.

Among the index alterations characterizing the genetic landscape of the lethal metastatic cell clone were mutations in PTEN (4-bp frameshifting deletion in exon 7 with loss of heterozygos- ity [LOH]), TP53 (R248Q, LOH), and SPOP (F133L), all known to be recurrent targets of mutation in advanced prostate cancer (12-14). These alterations were confirmed by Sanger sequencing in all 7 autopsy metastases (Figure 2A and Supplemental Figure 4). Immunohistochemical analysis showed that the PTEN and TP53 
A

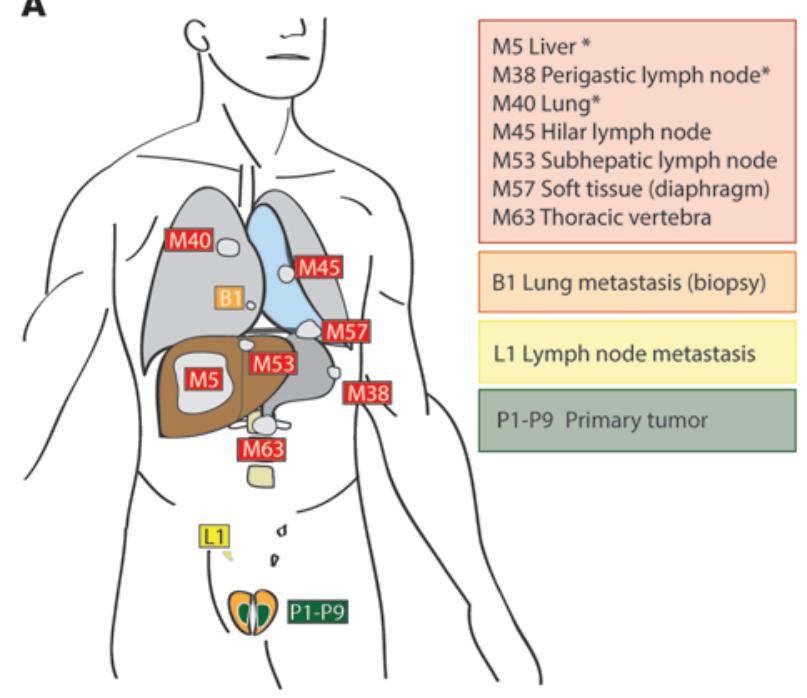

B

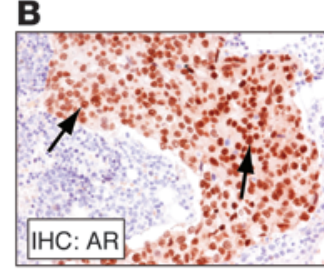

D

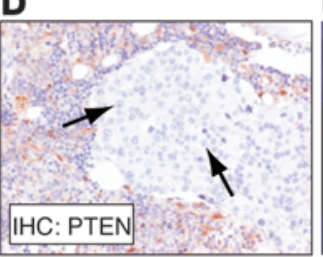

c

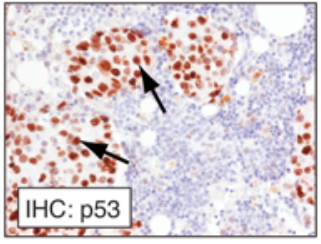

E

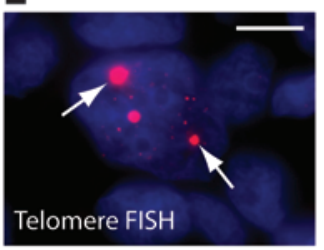

\section{Figure 2}

Consensus genomic alterations and their phenotypic consequences in the autopsy metastases. (A) Anatomic distribution of study samples. Asterisks denote the 3 anatomically distinct autopsy metastases on which whole-genome sequencing was performed. (B-E) Molecular phenotypes of genomic alterations evaluated by immunohistochemistry (IHC) and telomere-specific FISH in representative metastasis M63. (B) $A R$ amplification was associated with strong immunoreactivity for AR. (C) Mutations in TP53 (R248Q) resulted in nuclear accumulation of p53. (D) A frameshift deletion in the coding sequence of PTEN resulted in loss of PTEN immunostaining in neoplastic cells. Original magnification, $\times 20$. (E) The genomic inversion within the ATRX gene was associated with strong nuclear accumulation of telomeric sequence, consistent with ALT. Arrows indicate neoplastic cells. Scale bar: $10 \mu \mathrm{m}$.

mutations produced loss of PTEN staining and nuclear accumulation of p53, as expected (Figure 2, B-D, and Supplemental Figure 5). Thus, from a molecular taxonomy perspective, this case belongs to a prostate cancer subtype characterized by the absence of ETS rearrangement and the presence of SPOP mutations (12-14).

Interestingly, in all of the autopsy metastases, we detected an $18-\mathrm{Mb}$ inversion on chromosome $\mathrm{X}$ disrupting the ATRX gene, with associated loss of ATRX protein expression (Supplemental Figure 6). Loss-of-function alterations in ATRX are associated with massive intranuclear accumulation of telomeric DNA through alternative lengthening of telomeres (ALT) (15). Telomere-specific FISH showed telomeric aggregates, consistent with ALT, in all autopsy metastases (Figure 2E and Supplemental Figure 6). ATRX mutation may characterize a novel subgroup of metastatic prostate cancer; indeed, a recent study found $A T R X$ alterations in a small subset of metastatic prostate cancers (14).

To characterize the pathological landscape of the primary cancer, we comprehensively examined sections sampling the entire radical prostatectomy specimen (composed of 36 blocks), obtained more than 17 years prior (Supplemental Figure 1). The spectrum of morphologies and grades included small, focal areas of Gleason pattern 3, large areas of Gleason pattern 4, and foci of intraductal and ductal adenocarcinoma (Supplemental Figure 7). To identify primary cancer lesions sharing characteristics of the autopsy metastases, we first evaluated PTEN immunohistochemical staining. The vast majority of the primary cancer showed strong PTEN staining. Interestingly, we identified only a single small $(2.2 \mathrm{~mm} \times 1.3 \mathrm{~mm})$ lesion (referred to herein as P1), composed solely of Gleason pattern 3 tumor glands, that was devoid of PTEN staining in the primary cancer (Figure 3A and Supplemental Figures 7 and 8). Targeted sequencing of DNA recovered from microdissected cells from the PTEN-negative P1 revealed a 4-bp deletion in PTEN identical to the one present in the autopsy metastases (Figure 3B and Supplemental Figure 9). In contrast, this PTEN mutation was not present in DNA from 8 surrounding higher-grade lesions (P2-P9). Furthermore, the SPOP mutation was present in $\mathrm{P} 1$ as well as in $\mathrm{P} 6$ and P8, but not in any other sampled lesions from the primary cancer (Figure 3B and Supplemental Figure 9). Additionally, we detected the TP53 mutation in a subset of alleles from P1, but not from any other sampled region of the primary tumor (Figure 3B and Supplemental Figure 9), which suggests the emergence of a progressive subclone with TP53 mutation within P1. Together, these observations demonstrate a clonal relationship between P1 and the autopsy metastases and suggest that the lethal metastatic clone arose from P1 (a small, well-differentiated Gleason pattern 3 primary lesion), not from the prevalent Gleason pattern 4 cancer. This finding is particularly surprising since isolated Gleason pattern 3 lesions have shown no evidence of metastatic potential or progression to lethality $(16,17)$. Therefore, a Gleason pattern 3 lesion in close proximity to higher-grade lesions could have biological properties different than those of isolated Gleason pattern 3 lesions. Furthermore, because P1 was the only part of the primary cancer containing cells with index mutations in PTEN and TP53, which have previously been associated with aggressive disease (18-20), comprehensive evaluation of PTEN and TP53 status could be useful for identifying lesions in the primary tumor that are more likely to progress. Overall, these data suggest that P1 initially seeded a micrometastasis that escaped initial therapy and gave rise to all subsequent metastases, either directly or indirectly, through sequential dissemination from metastasis to metastasis.

Despite the similarities between P1 and the autopsy metastases, we observed evidence for additional clonal evolution. None of the primary lesions, including $\mathrm{P} 1$, harbored the ATRX rearrangement or $A R$ amplification (Figure 3B and Supplemental Figures 3 and 6), 
A
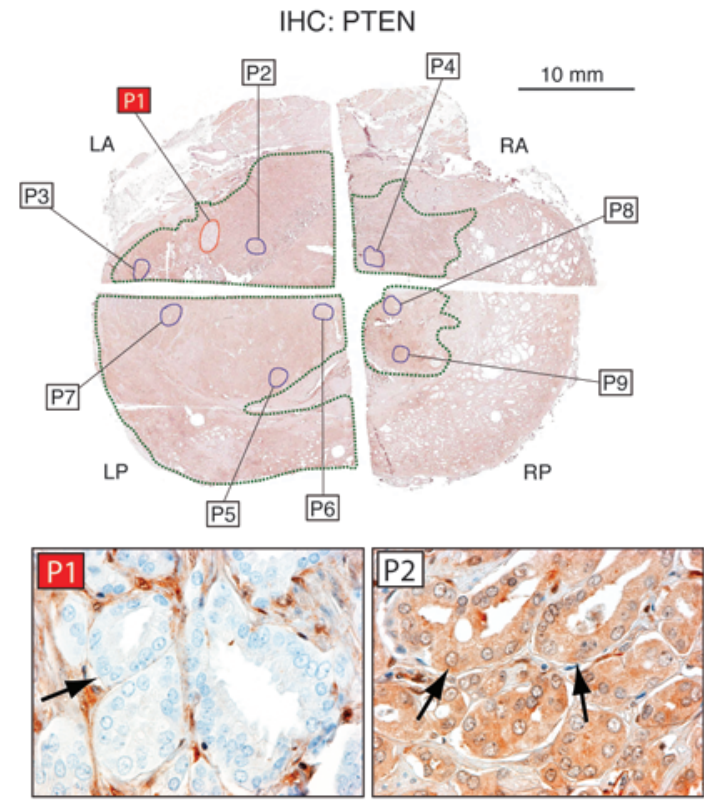

B

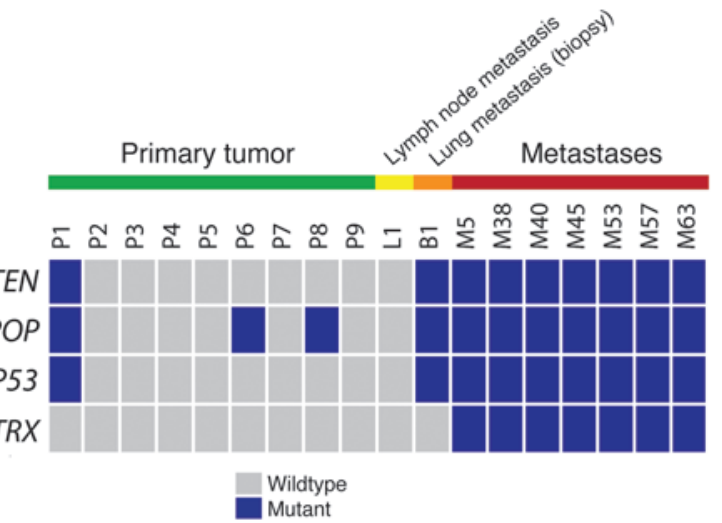

C

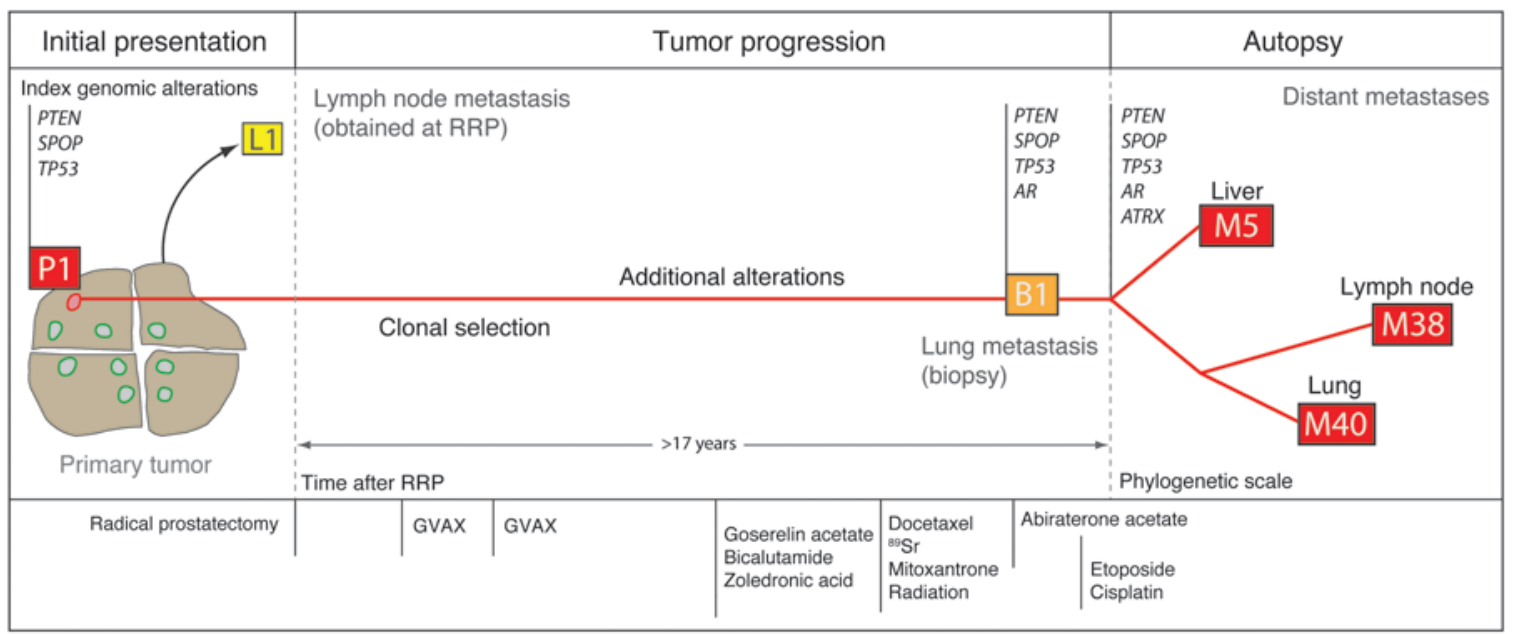

\section{Figure 3}

Molecular and pathological findings in the primary tumor and their clonal relationship to the distant metastases. (A) PTEN staining in a cross-section of the primary prostatectomy specimen (LA, left anterior; RA, right anterior; LP, left posterior; RP, right posterior). Individual tumor areas that were further analyzed are indicated; green dotted outline denotes areas containing tumor glands. P1 was the only lesion in the primary tumor devoid of PTEN staining in neoplastic cells. The adjacent P2 stained positive for PTEN, and in this regard was representative of the bulk tumor outside P1. Arrows indicate tumor cells. Note that the surrounding normal stroma showed strong immunoreactivity for PTEN. Original magnification, $\times 2$ (top); $\times 40$ (bottom). Scale bar: $10 \mathrm{~mm}$. (B) Summary of the analyzed consensus genomic alterations in the primary tumor and metastases. The presence and absence of the consensus mutations are denoted by blue and gray, respectively. (C) Proposed model of disease progression in this index case, based on sequencing and molecular pathological analyses. Phylogenetic relationships of distant metastases were calculated based on structural rearrangements.

which suggests that generation and selection of a cell clone harboring these alterations was a later event, likely arising after androgen deprivation therapy (Supplemental Figure 3). Furthermore, a lung lesion that was biopsied 16 months prior to autopsy showed no evidence for ALT and ATRX alterations, despite having the PTEN, TP53, and SPOP mutations, amplification of $A R$, and high proliferation rates (Ki-67 index, >25\%) similar to those of the autopsy metastases (Figure 3 and Supplemental Figures 9 and 10). This indicates that the ATRX alteration may represent a very late event in this case.

Interestingly, the lymph node metastasis resected at radical prostatectomy did not harbor PTEN, SPOP, TP53, or ATRX muta- tions (Supplemental Figures 9 and 10), suggestive of an independent clonal/subclonal origin of this lesion. This finding provides proof-of-concept of the potential utility of repeated longitudinal evaluation of lesions during clinical management in order to effectively target the evolving spectrum of molecular alterations during progression (21-24). These observations also suggest that multiple tumor clones may arise, regress, and evolve during disease progression and treatment, similar to what has been observed for other cancers (21-24). However, the degree of clonal heterogeneity within the primary tumor and between the primary tumor and distant metastases may vary significantly in different tumor 
types $(22,24,25)$. Additionally, the various therapies, including the experimental GVAX vaccine, aggressive androgen ablation, or the other interventions, may have substantially affected the clonal evolution of the disease in this case (Figure 3C). For instance, inactivation of PTEN has been associated with resistance to androgen ablation (26), which might explain the clonal selection of the PTEN mutated cell clone during hormonal therapy in this case.

A limitation of this study is that, since this is the first prostate cancer case for which it was possible to carry out such detailed longitudinal characterization of the lethal cell clone from the primary cancer to distant metastases, the extent to which these findings are generalizable is unclear. Nonetheless, this case is in many regards highly typical of prostate cancer in a clinical and molecular sense, which may indicate that these findings could be more generalizable. Future studies with additional cases will be needed to test this notion. Another limitation is that microdissection of the primary tumor lesions from archival FFPE blocks that were approximately 2 decades old yielded insufficient high-quality DNA for genome-wide analyses. This limited our ability to comprehensively delineate potential subclonal relationships between P1 and other lesions in the primary cancer and metastases. For example, such analyses could have allowed delineation of a possible subclonal relationship among P1, P6, and P8, all of which harbored the recurrent SPOP mutations.

Broadly, our present study documents the potential of integrated genomic and histopathological approaches to characterize tumor heterogeneity. It also provides proof-of-concept of the potential importance of molecular staging and grading strategies, in conjunction with existing pathological criteria, to accurately inform clinical decision making in precision medicine. Using such longitudinal evaluation, future efforts to examine clonal evolution in prostate cancer progression can help us understand whether this index case represents a rare outlier or a common manifestation of the disease.

\section{Methods}

7 distant metastases from distinct anatomic sites were procured by rapid autopsy. DNA of 3 metastases and normal constitutional DNA was subjected to massively parallel whole-genome sequencing by Complete Genomics (11). Archival FFPE samples of the primary tumor, a lymph node metastasis resected at radical prostatectomy, and a biopsy of the lung metastasis were enriched for tumor cells by microdissection, and extracted DNA was analyzed by PCR and Sanger sequencing. Immunohistochemistry for PTEN, p53, and ATRX and telomere-specific FISH were performed as described previously $(15,20)$. See Supplemental Methods and Supplemental Table 5 for details.

Study approval. The Johns Hopkins Institutional Review Board approved the study, and written informed consent was obtained.

\section{Acknowledgments}

This manuscript is dedicated to the gentleman and his family, whose commitment to advancing prostate cancer research and generous tissue donation made this study possible. This study was supported by funding from the NIH/NCI (P50CA058236, R01CA070196); DOD (PC073533/W81XWH-08-1-0049); The Prostate Cancer Foundation David Mazzone Challenge award and Creativity award; The V Foundation Martin D. Abeloff V Scholar Award (to S. Yegnasubramanian); The Cleveland Foundation Ellen B. Masenhimer Fellowship (to S. Yegnasubramanian); and the Commonwealth Foundation for Cancer Research. Alison Mass-Bommarito, Sal Bommarito, and the Irving Hansen Foundation provided additional generous philanthropic support. M.C. Haffner is supported by the Richard and Ellen Sandler Young Investigator Award from the Prostate Cancer Foundation. This work is supported by the Department of Defense Prostate Cancer Research Program, DOD award no. W81XWH-10-2-0056 and W81XWH-10-2-0046 PCRP Prostate Cancer Biorepository Network (PCBN).

Received for publication April 9, 2013, and accepted in revised form August 12, 2013.

Address correspondence to: Srinivasan Yegnasubramanian, Sidney Kimmel Comprehensive Cancer Center, Johns Hopkins University, Koch Cancer Research Bldg., Rm. 145, 1550 Orleans St., Baltimore, Maryland 21231, USA. Phone: 410.502.3425; Fax: 410.502.9817; E-mail: syegnasu@jhmi.edu.
1. Siegel R, Naishadham D, Jemal A. Cancer statistics, 2012. CA Cancer J Clin. 2012;62(1):10-29.

2. Wilt TJ, et al. Radical prostatectomy versus observation for localized prostate cancer. $N$ Engl J Med. 2012;367(3):203-213.

3. Chou R, LeFevre ML. Prostate cancer screening the evidence, the recommendations, and the clinical implications. JAMA. 2011;306(24):2721-2722.

4. Arora R, Koch MO, Eble JN, Ulbright TM, Li L, Cheng L. Heterogeneity of Gleason grade in multifocal adenocarcinoma of the prostate. Cancer. 2004; 100(11):2362-2366.

5. Clark J, et al. Complex patterns of ETS gene alteration arise during cancer development in the human prostate. Oncogene. 2008;27(14):1993-2003.

6. Lindberg J, et al. Exome sequencing of prostate cancer supports the hypothesis of independent tumour origins. Eur Urol. 2012;63(2):347-353.

7. Liu W, et al. Copy number analysis indicates monoclonal origin of lethal metastatic prostate cancer. Nat Med. 2009;15(5):559-565.

8. Mehra R, et al. Characterization of TMPRSS2ETS gene aberrations in androgen-independent metastatic prostate cancer. Cancer Res. 2008; 68(10):3584-3590.

9. Osler W. The Principles and Practice of Medicine. 1st ed. New York, New York, USA: D. Appleton and Company; 1893
10. Simons JW, et al. Phase I/II trial of an allogeneic cellular immunotherapy in hormone-naive prostate cancer. Clin Cancer Res. 2006;12(11 pt 1):3394-3401.

11. Drmanac R, et al. Human genome sequencing using unchained base reads on self-assembling DNA nanoarrays. Science. 2010;327(5961):78-81.

12. Barbieri CE, et al. Exome sequencing identifies recurrent SPOP, FOXA1 and MED12 mutations in prostate cancer. Nat Genet. 2012;44(6):685-689.

13. Berger MF, et al. The genomic complexity of primary human prostate cancer. Nature. 2011; 470(7333):214-220

14. Grasso CS, et al. The mutational landscape of lethal castration-resistant prostate cancer. Nature. 2012;487(7406):239-243.

15. Heaphy CM, et al. Altered telomeres in tumors with ATRX and DAXX mutations. Science. 2011; 333(6041):425.

16. Hernandez DJ, et al. Natural history of pathologically organ-confined (pT2), Gleason score 6 or less, prostate cancer after radical prostatectomy. Urology. 2008;72(1):172-176.

17. Ross HM, Kryvenko ON, Cowan JE, Simko JP, Wheeler TM, Epstein JI. Do adenocarcinomas of the prostate with Gleason score (GS) $\leq 6$ have the potential to metastasize to lymph nodes? Am J Surg Pathol. 2012;36(9):1346-1352.

18. Markert EK, Mizuno H, Vazquez A, Levine AJ.
Molecular classification of prostate cancer using curated expression signatures. Proc Natl Acad Sci US A. 2011;108(52):21276-21281.

19. Chen $Z$, et al. Crucial role of p53-dependent cellular senescence in suppression of Pten-deficient tumorigenesis. Nature. 2005;436(7051):725-730.

20. Lotan TL, et al. PTEN protein loss by immunostaining: analytic validation and prognostic indicator for a high risk surgical cohort of prostate cancer patients. Clin Cancer Res. 2011;17(20):6563-6573.

21. Ding $\mathrm{L}$, et al. Clonal evolution in relapsed acute myeloid leukaemia revealed by whole-genome sequencing. Nature. 2012;481(7382):506-510.

22. Gerlinger $M$, et al. Intratumor heterogeneity and branched evolution revealed by multiregion sequencing. N Engl J Med. 2012;366(10):883-892.

23. Mardis ER. Genome sequencing and cancer. Curr Opin Genet Dev. 2012;22(3):245-250.

24. Ding $\mathrm{L}$, et al. Genome remodelling in a basal-like breast cancer metastasis and xenograft. Nature. 2010;464(7291):999-1005.

25. Yachida S, et al. Distant metastasis occurs late during the genetic evolution of pancreatic cancer. Nature. 2010;467(7319):1114-1117.

26. Carver BS, et al. Reciprocal feedback regulation of PI3K and androgen receptor signaling in PTEN-deficient prostate cancer. Cancer Cell. 2011; 19(5):575-586. 